\title{
Research on Window Function Selection in Phase Estimation of Digital Signal
}

\author{
He Jing, Liu Yang \\ China Satellite Maritime Tracking and Control Department, Jiangyin, 214431, China
}

Keywords: window function; estimated accuracy; window length

\begin{abstract}
In the process of phase estimation of digital signal, windowing will cause the problem of spectrum leakage. In view of this problem, this paper studies the solution of using appropriate window function to reduce spectrum leakage. And then analyzes the influence of different window functions and window length on the phase estimation accuracy.
\end{abstract}

\section{Introduction:}

The spectrum after energy leakage is determined by the window shape corresponding to the window function used. Therefore, the appropriate analysis window function should be selected to achieve the best demodulation performance.

\section{Effect of different window functions on phase estimation accuracy}

As a result of the windowing of the signal, the lower resolution and the occurrence of leakage are two major effects on the spectrum. The resolution is mainly affected by the width of the main lobe of the window function Fourier transform, and the degree of leakage depends on the relative amplitude of the main lobe and sidelobe of the window function Fourier transform. In DSTFT-based demodulation, because the DQPSK signal is a single frequency signal, so the frequency domain resolution is not high, and the frequency domain phase accuracy requirements are higher, so should choose the main lobe width slightly wider, with a smaller side Flap peak and larger sidelobe peak attenuation rate of the window function.

Signal processing commonly used window function rectangular window, triangular window, Hanning window, Hamming window, Blackman window, Catherine window, Chebyshev window and so on. Based on the analysis of the spectral characteristics of various window functions, the generalized cosine window is more suitable for the window function than the DSTFT demodulation algorithm if the tunable window and the Chebyshev window are not considered.

The computer simulation produces 100 sinusoidal signals with a frequency of $2.1 \mathrm{MHz}$ and a sampling rate of $16 \mathrm{MHz}$. The initial phase takes a different value. The mean phase estimation of the 100 sinusoidal signals is given using several of the window functions mentioned above. The mean values of the statistical phase estimation errors are shown in Table 1 and Table 2.

Table 1. Sine Signal Phase Estimation Mean Error $(\mathrm{M}=8)$

\begin{tabular}{|l|l|l|l|l|l|}
\hline $\begin{array}{l}\text { Window } \\
\text { Function }\end{array}$ & $\begin{array}{l}\text { Rectangular } \\
\text { window }\end{array}$ & $\begin{array}{l}\text { Triangle } \\
\text { window }\end{array}$ & $\begin{array}{l}\text { Hanning } \\
\text { window }\end{array}$ & $\begin{array}{l}\text { Hamming } \\
\text { window }\end{array}$ & $\begin{array}{l}\text { Blackman } \\
\text { window }\end{array}$ \\
\hline Mean $(|\Delta|)$ & 0.034469 & 0.023017 & 0.0012347 & 0.024916 & 0.015415 \\
\hline \multirow{2}{*}{$\begin{array}{l}\text { Window } \\
\text { Function }\end{array}$} & \multicolumn{3}{|c|}{$\begin{array}{l}\text { Chebyshev window } \\
\text { (First sidelobe versus main lobe attenuation } \\
/ \mathrm{dB})\end{array}$} & $\begin{array}{l}\text { Catherine window } \\
\text { (First sidelobe versus main lobe } \\
\text { attenuation / dB) }\end{array}$ \\
\hline \multirow{2}{*}{ Mean $(|\Delta|)$} & \multicolumn{2}{|c|}{\begin{tabular}{c}
$0.00092986(100 \mathrm{~dB})$ \\
\cline { 2 - 4 }
\end{tabular}} & \multicolumn{2}{|c|}{$0.032113(50 \mathrm{~dB})$} & $0.06567(100 \mathrm{~dB})$ \\
\cline { 2 - 5 } & \multicolumn{2}{|c|}{$0.067244(30 \mathrm{~dB})$} & $0.068293(50 \mathrm{~dB})$ \\
\hline
\end{tabular}


Table 2. Sine Signal Phase Estimation Mean Error $(M=16)$

\begin{tabular}{|c|c|c|c|c|c|}
\hline $\begin{array}{l}\text { Window } \\
\text { Function }\end{array}$ & $\begin{array}{l}\text { Rectangular } \\
\text { window }\end{array}$ & $\begin{array}{l}\text { Triangle } \\
\text { window }\end{array}$ & $\begin{array}{l}\text { Hanning } \\
\text { window }\end{array}$ & $\begin{array}{l}\text { Hamming } \\
\text { window }\end{array}$ & $\begin{array}{l}\text { Blackman } \\
\text { window }\end{array}$ \\
\hline Mean $(|\Delta|)$ & 0.0329 & 0.0011966 & $6.6747 * 10$ & 0.0055498 & $1.4897 * 10^{-4}$ \\
\hline $\begin{array}{l}\text { Window } \\
\text { Function }\end{array}$ & \multicolumn{3}{|c|}{$\begin{array}{c}\text { Chebyshev window } \\
\text { (First sidelobe versus main lobe attenuation } \\
\qquad / \mathrm{dB} \text { ) }\end{array}$} & \multicolumn{2}{|c|}{$\begin{array}{c}\text { Catherine window } \\
\text { (First sidelobe versus main lobe } \\
\text { attenuation } / \mathrm{dB} \text { ) }\end{array}$} \\
\hline \multirow{3}{*}{ Mean $(|\Delta|)$} & \multicolumn{3}{|c|}{$6.63389 * 10^{-6}(100 \mathrm{~dB})$} & \multicolumn{2}{|c|}{$5.683 * 10^{-5}(100 \mathrm{~dB})$} \\
\hline & \multicolumn{3}{|c|}{$2.8159 * 10^{-5}(50 \mathrm{~dB})$} & \multicolumn{2}{|c|}{$0.011171(50 \mathrm{~dB})$} \\
\hline & \multicolumn{3}{|c|}{$0.01488(30 \mathrm{~dB})$} & \multicolumn{2}{|c|}{$0.064299(30 \mathrm{~dB})$} \\
\hline
\end{tabular}

$\mathrm{M}$ in the table is the length of the window function, that is, the number of samples after the truncated signal. It can be seen from the mean value of the statistical error that the initial phase estimation error of the generalized cosine window is small, and if the window length is longer, the initial phase estimation accuracy of the Chebyshev window and the Catherine window is higher when the sidelobe decay is faster. The results show that the generalized cosine windows (especially the Hanning windows), as well as the phase estimation performance of the Chebyshev window and the Catherine window, are better.

\section{The Effect of Window Length on Phase Estimation Accuracy}

From the previous simulation has been able to see the window length of the choice of phase estimation will also have an impact, it needs to be based on data indicators and demodulation requirements for reasonable settings. Assuming that the length of the window function is $M$, the number of sampling points included in a carrier cycle is $\mathrm{K}$, the number of sampling points in a double bit code is $\mathrm{L}$, and the selection of $\mathrm{M}$ in DSTFT demodulation takes into account the following two aspects:

A). To ensure the accuracy of each measurement, the window length is at least one carrier cycle width, that is, $M \geqslant K$.

B). Due to the phase transition caused by the symbol shift, when the window from a symbol slip into the next symbol before the phase estimation is accurate, that is, a symbol can be accurately obtained before (LM) sampling point phase , To ensure a sufficient amount of decision, need LM> $\mathrm{L} / 2$, which has $\mathrm{M}<\mathrm{L} / 2$.

Therefore, the selection of $\mathrm{M}$ must meet the following conditions:

$$
K \leq M<\frac{L}{2}
$$

The experimental basis for supporting this conclusion is given by computer simulation.

\section{Simulation test}

Influence of Test Window Length on Initial Phase Estimation Error of DFT Based Sine Signal. The frequency of the sinusoidal signal is $2.1 \mathrm{MHz}$ and the sampling rate is $16 \mathrm{Msps}$. As shown in Fig. 1 , the sine signal of Hanning window, Chebyshev window and Catherine window are taken at different lengths.

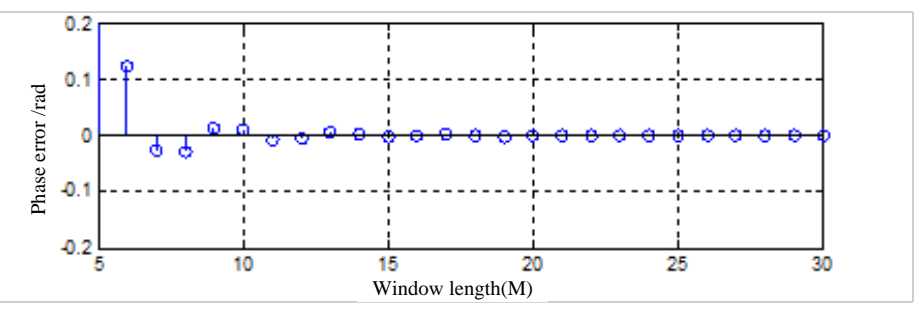

(a) Hanning window 


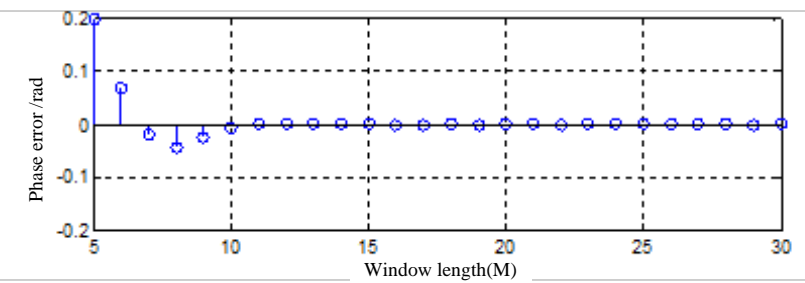

(b) Chebyshev window

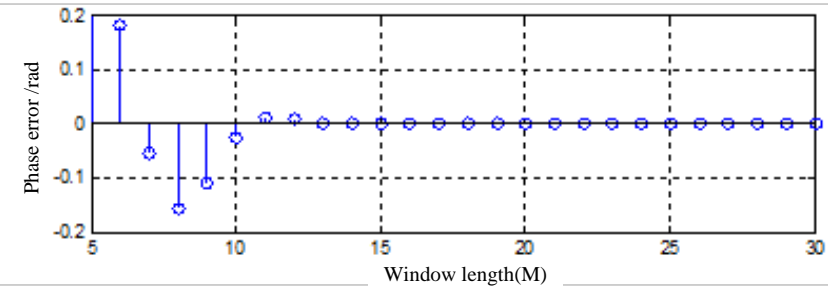

(c) Catherine window

Figure 1. Effect of window length on phase measurement

According to the signal parameters, at this time a carrier cycle contains seven sampling points, we can see from Figure 1, when $\mathrm{M}<7$, the three window function of the phase error are larger, and $M \geqslant 10$ when the phase The error has been very small. It also shows that the window length should contain at least one carrier period of the sampling data.

When the test window takes different lengths, the DSTFT algorithm is used to estimate the instantaneous phase estimation of the DQPSK signal. Figure 2 shows the phase error of the Hanning window in various window lengths. The bandpass Gaussian white noise is added to the signal. The noise ratio is $12.8 \mathrm{~dB}$.

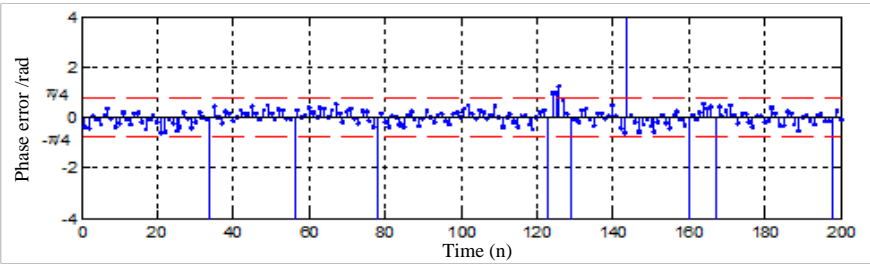

(a) Window length $M=6$

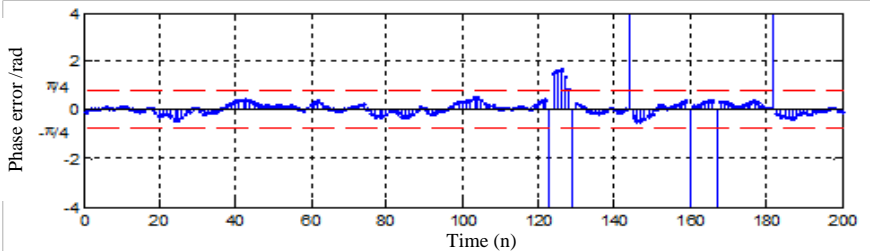

(b) Window length $M=8$

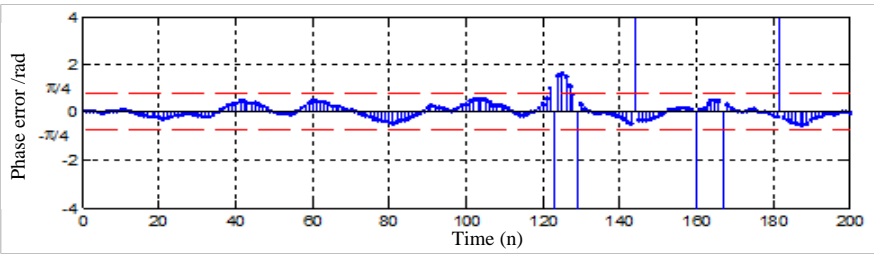

(c) Window length $\mathrm{M}=10$

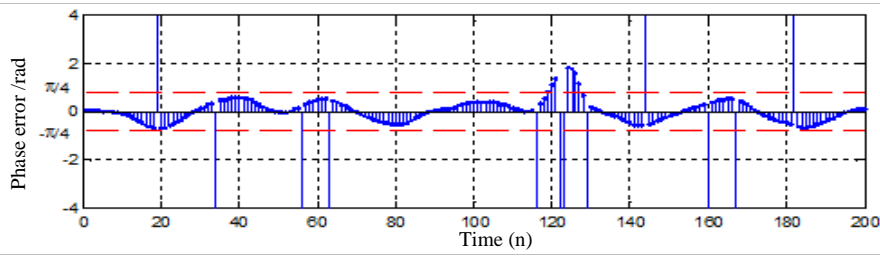

(d) Window length $M=16$

Figure 2. Hinging window instantaneous phase estimation error

Figure 2 shows the use of Hanning window on the DQPSK signal instantaneous phase estimation error, the window length of 6,8,10,16 respectively. Since the interval between the decision 
thresholds in the DSTFT algorithm is $\pi / 2$, the phase error is not caused if the absolute value of the phase error is less than $\pi / 4$. In order to facilitate the measurement of the error range, the figure marked $\pi / 4$ and $-\pi / 4$ two error limits, as long as the phase error between the two error limits, it will not affect the demodulation.

It is noted that there are several points with large phase error values in Fig. 2, not because the phase is not accurate but is caused by the modulo $2 \pi$ operation at the time of calculation. For example, if the actual phase is 6.24rad, the estimated phase is 6.32rad, and the error calculated by the phase error is:

$$
\Delta \theta=\theta_{0}-\bmod (\hat{\theta})=6.24-0.0368=6.2036(\mathrm{rad})
$$

While the actual error is only 6.24-6.32 $=0.08 \mathrm{rad}$.

The influence of the length of the discussion window on the phase estimation of the DQPSK signal is analyzed with reference to Fig. 2, where the number of sampling points in one carrier period is equal to 7 and the number of sampling points in a double bit code is equal to 21 . First, $\mathrm{M}=$ 6 does not meet $M \geqslant K$, from Figure 2 (a) can be seen when the phase error is large. $M=8$ and $M$ = 10 can satisfy $M \geqslant K$ and $M$ \& lt; $L / 2$ at the same time, so Fig. 2 (b) and Fig. 2 (c) show that the overall phase measurement performance is better. $\mathrm{M}=16$, the exact phase of a symbol can only be $L M=5$, and most of the sampling point phase estimation error is too large, from Figure 2 (d) can be seen that its overall phase measurement performance is poor, Thus proving the necessity of condition $\mathrm{M}<\mathrm{L} / 2$. The correctness of the principle of the selection of the window function length $\mathrm{K} \leqslant \mathrm{M}<\mathrm{L} / 2$ is verified by the above two simulation tests.

\section{Summary}

In this paper, the problem of spectral leakage caused by window truncation is discussed, and the solution of using appropriate window function to reduce spectrum leakage is studied. And then analyzes the influence of different window functions and window length on the phase estimation accuracy.

\section{Reference:}

[1] L.Erup, F,M,Gardner, interpolation in digital modems-part II. Implementation and performance, IEEE. Trans. Com, Jun.2013, 41(6):998-1008.

[2] Chuang J.C, Sollenberger N.R, Burst coherent demodulation with combined symbol timing frequency offset estimation, and diversity selection. IEEE,Trans.Com, 2011, 39(7):1157-1164.

[3] Sollemberger N.R, Chuang J.C, Low Overhead symbol timing and carrier recovery for TDMA portable radia systems. IEE, Trans.Com, 2010, 38(10):1886-1892. 